COMPTES RENDUS

\title{
DE LA RÉFORME AUX GUERRES DE RELIGION
}

Denis Crouzet, La Genèse de la Réforme française. 1520-1562. Paris, SEDES, 1996. $17,5 \times 24,620$ p. (Regards sur l'histoire).

La Genèse de la Réforme française, 1520-1562, tout en étant au départ destinée aux agrégatifs d'histoire, s'inscrit résolument dans l'œuvre de Denis Crouzet. Cet ouvrage est à la fois la mise au point de l'un des meilleurs spécialistes actuels de ces questions et un ouvrage pédagogique aux vues originales, dont la principale est la grande espérance eschatologique des réformés français. Sa construction reflète la conviction de l'auteur : l'histoire est « un immense champ heuristique, et donc un lieu de virtualités et de contradictions ». Ainsi l'ouvrage vise-t-il à proposer non seulement la réflexion de l'auteur mais également les principales thèses en présence, en vertu de la pluralité des interprétations possibles d'un même événement. Certes, il existe pour lui des versions plus plausibles que d'autres, et son ordre de présentation suit en conséquence un ordre croissant, lui-même intervenant souvent en dernier. La genèse de la Réforme française est étudiée en cinq chapitres, suivant un plan chronologique. Le premier expose le « cas Luther », selon l'expression de Jean Delumeau, et le «cas Lefèvre d'Étaples »; le deuxième se concentre sur l'évangélisme; le troisième sur Calvin; le quatrième sur la diffusion progressive du calvinisme; enfin, le cinquième chapitre présente les différentes thèses tentant d'expliquer les adhésions à la Réforme en France jusqu'en 1562, la genèse prenant fin avec l'édit du 17 janvier 1562 qui accorde aux réformés la liberté de culte (hors des villes closes, de jour seulement, sans port d'armes).

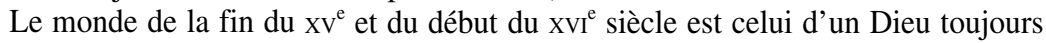
présent, soit miséricordieux, soit rappelant à l'ordre par le biais de mirabilia. Mais à partir de 1517, tous les messages divins sont liés à l'entrée en scène de Martin Luther. C'est donc en premier lieu sur la rupture opérée par le docteur de Wittenberg que Crouzet se concentre, en rappelant que Luther a réécrit sa vie et présenté sa réflexion de manière peut-être beaucoup plus évolutive qu'elle ne l'a été. Car le plus important (le salut par la justice de Dieu précède les œuvres) est déjà fixé avant 1517. L'auteur étudie la doctrine luthérienne et le "déphasage » qu'elle subit dans les controverses : tout se met à tourner autour de l'ecclésiologie sous le fait de la pression romaine alors que, selon lui, qui s'appuie sur les recherches de Heiko A. Oberman, «Luther avant d'être le destructeur d'une construction ecclésiologique

Revue de synthèse : $5^{\mathrm{e}}$ série, année 2005/1, p. 161-264. 
est le destructeur d'un système sotériologique » (p. 45). Luther rompt avec un univers mental d'angoisse devant la mort (p. 45-66). L'auteur relève les indices qui révèlent une sensibilité religieuse pour laquelle le moment précédant la mort est central. Or la mort pour Luther est délivrance. Il n'y a pas de jugement dans l'Audelà (p. 67). «Le jugement est avant», selon l'expression de Pierre Chaunu. La révolution luthérienne «détruit l'angoisse de la mort mais maintient l'angoisse eschatologique » (p. 68). C'est seulement avec Calvin que le désangoissement sera parachevé. Cependant, la réception de la «révolution» luthérienne en France doit être nuancée. En étudiant la diffusion des idées de Luther, Crouzet reprend la démonstration de Robert J. Knecht (Francis I, Cambridge, 1982) : les autorités ne réagissent pas de la même façon car, tandis que le roi lutte contre Luther, la Sorbonne concentre son action contre les humanistes (p. 86-87).

Dans l'étude du «cas Lefèvre d'Étaples », «théologien de la "synergie" de la grâce et de la liberté » (p. 95) sont soulignées les particularités par rapport à Luther (comme ce dernier, il estime la fin des Temps proche mais ne devant advenir que lors de la «réunion des fidèles dans le culte de l'Évangile ») et à Érasme (Lefèvre préfère un Christ métaphysique à un Christ souffrant, « crucifié couronné d'épines sanglantes », et sa quête est plus celle «d'une sainteté mystique » que d'une «philosophia Christi» accessible à tous). Enfin, Lefèvre, selon Augustin Renaudet, «jugeait encore le culte romain compatible avec le culte spirituel, si les fidèles vivaient réellement en esprit » (p. 102). Le chapitre se clôt sur le cas de Guillaume Briçonnet, évêque de Meaux, à la sensibilité religieuse optimiste et providencialiste, et montre à partir de la démonstration de Claude Blum (p. 108-110) que son diocèse «fonctionne un peu comme le laboratoire de l'imaginaire » que tente d'instituer le concordat de Bologne (p. 113).

Le deuxième chapitre porte sur «la période de détermination incertaine des partages religieux, qui s'étend jusqu'en $1535 »$. Crouzet souligne que la Réforme française «ne peut se comprendre uniquement dans une construction oppositionnelle par rapport à l'Église établie ». Il étudie le réformisme du cercle des bibliens dans le diocèse de Meaux, dont l'ambition première est un Nouveau Testament accessible à tous les fidèles. Le caractère sacré de la mission des clercs est amplifié. L'exemple, développé par Thierry Wanegffelen, de Pierre Caroli, protégé de Marguerite de Navarre qui erre entre la France et Genève, tour à tour catholique ou réformé, montre «qu'il y a des hommes pour qui il est impossible [...] de se reconnaître dans le processus de [...] confessionnalisation qui est en gestation» (p. 157). L'auteur complète cette histoire d'un « christianisme non confessionnel », selon l'expression de Wanegffelen, en mettant en évidence "l'immense espérance en une réalisation du Christ » qui le sous-tend. « Ce qui caractérise les bibliens, par rapport aux luthériens comme aux catholiques romains, ce serait [...] une focalisation de la foi vers la Parole de Dieu et une tendance à minimiser, voire occulter les traditions humaines » (p. 159). Il met en évidence les évolutions profondes qui «raidissent » l'évangélisme jusqu'à la dispersion du groupe de Meaux (p. 178-237). En parallèle, il étudie l'attitude de la monarchie face aux «luthériens », dont le refus de l'eucharistie remet en cause l'idéologie même de la monarchie fondée sur une dimension eucharistique de la personne royale. Cette opposition se cristallise lors des deux affaires des Placards (p. 229-237). Cependant, selon Crouzet, François $I^{\mathrm{er}}$ ne renonce jamais à une réunion des Églises. 
Le troisième chapitre s'arrête sur le cas Calvin en proposant les quatre thèses qui s'opposent sur sa conversion : celle de François Wendel, de Pierre Chaunu, de l'américain William J. Bouwsma et de Bernard Cottret (p. 242-246). De ce dernier, l'auteur retient cette ambition : l'historien doit « essayer de découvrir Calvin dans le mouvement même d'une autobiographie ». Après avoir étudié le parcours plutôt commun de Jean Calvin jusqu'en 1536, il se consacre à l'étude de l'Institution de la religion chrétienne (p. 253-258). Puis il s'interroge sur ce qui oppose le «système calvinien » à celui de Luther : alors que, pour ce dernier, « le don gratuit de la foi » est reçu lorsque l'homme prend conscience de sa déchéance, pour Calvin c'est « la perception de la majesté de Dieu » qui fait comprendre à l'homme son état de pécheur, préalable incontournable à «la suivie de la sagesse de Dieu» (p. 270). Quant aux évangéliques, Calvin s'en distingue, selon Chaunu et Blum, en affirmant que «l'homme demeure dans l'ordre du péché par delà la Rédemption [...] et [que] c'est le Christ, seul, qui sauve » (p. 273). Car la « souveraineté de Dieu » entre 1534 et 1562 est loin d'être uniquement calvinienne. Crouzet se penche sur la spiritualité des évangéliques : ainsi Rabelais, contrairement à Luther et à Calvin, croit en la coopération de l'homme et de Dieu et ne voit pas en l'homme un «bénéficiaire passif » (p. 328).

Le quatrième chapitre étudie la progressive domination par Calvin de la spiritualité hétérodoxe en France. En premier lieu, sont retracés les «chemins de l'hérésie », expression forgée par Eugénie Droz : l'imprimé, la parole, la violence iconoclaste, «pédagogie destinée à dire la corruption de la piété qu'est la religion papiste », comme l'a montré Olivier Christin (p. 376). L'auteur souligne également la colossale entreprise d'impression que représente l'établissement du Psautier. À la diffusion de la doctrine calviniste répond une répression dont le profil se modifie : Crouzet établit une chronologie précise (p. 398-399) et étudie la "sécularisation » du crime d'hérésie, la censure (pour laquelle collabore faculté de théologie et monarchie) et la «chasse aux paroles ». La collaboration entre la monarchie et la Sorbonne culmine en 1543 dans l'élaboration d'un credo catholique, dirigé contre les calvinistes et destiné à clore la sphère de la piété (p. 404), credo auquel Calvin s'empresse de répondre. L'inégalité spatiale de la répression (p. 406-412) est soulignée : l'auteur rappelle que les autorités prennent parfois conscience que le martyre peut être aussi risqué que la clémence. Alors que du côté catholique les mises à mort se veulent des anticipations du châtiment infernal, la mort chez les réformés est une libération et le martyre, un «signe de victoire imminente » (p. 433). Le calvinisme s'impose donc comme une force de plus en plus importante, non seulement face aux autorités catholiques mais aussi face aux autres voies hétérodoxes que la prise de contrôle calvinienne des communautés de prières va faire disparaître. Avec l'éviction des paroles « impures », impures selon la tradition genevoise, avec la lutte contre la prise de parole des anabaptistes, des libertins spirituels, des défroqués, s'est déroulée selon Crouzet la destruction de tout un univers religieux pluriel qu'on ne connaîtra jamais plus que par bribes. Mais le remplacement des communautés de prières par des Églises dressées, le choix de l'institutionnalisation vient d'une pression de la base (p.452), d'un « désir de cène»(p. 457). L'extension croissante du mouvement, notamment par le biais d'adhésions nobiliaires, aboutit à la tenue du premier synode national (mai 1559) dont l'auteur expose la confession de foi, la discipline ecclésiastique et les mises en application (p. 465-469). À la fin 
du chapitre est soulignée la tension régicide présente dans la dynamique calviniste des temps des troubles de religion.

Le cinquième et dernier chapitre propose une mise en perspective de différentes hypothèses qui visent à expliquer le choix d'une adhésion à la Réforme. L'auteur analyse d'abord l'hypothèse soulignant les conditions socio-économiques d'une conversion à partir du modèle Hauser (équation Réforme = pauvreté) et ses critiques les plus récentes. Puis il passe en revue les thèses plaçant en position dominante la matrice socioculturelle, en exposant entre autres la thèse de Mack P. Holt sur la non-réception des idées huguenotes par les vignerons motivée par l'adéquation des rites de cette communauté avec les rites catholiques (p. 508-509). Une troisième voie interprétative insiste soit sur le poids du milieu géohistorique (par exemple, les différentes « Normandies » étudiées par David Nicholls), soit sur celui des spécificités locales : il s'agit de porter l'attention sur les conditions particulières à chaque espace d'essor des idées nouvelles (p. 523). Enfin, une dernière tendance historiographique se préoccupe de l'«adéquation Réforme et politique », en s'appuyant sur des monographies régionales, telle celle sur le Limousin par Michel Cassan (p. 527-546). Puis trois interprétations de l'engagement politique nobiliaire sont présentées : le «devoir de révolte » (Arlette Jouanna), la stratégie d'avancement personnel, le besoin sacral de guerre, la guerre étant vécue comme une rencontre avec Dieu (p. 539).

L'ouvrage se referme sur une période de grande exaltation de la part des réformés. Le « règne de l'Évangile » paraît imminent. L'échec de la répression est patent dans les années 1560-1562. Il mène à une tentative de « décriminalisation de l'hérésie » (p. 572-592) qui trouve son aboutissement dans l'édit de janvier 1562. Crouzet clôt ici sa genèse, une autre histoire commence.

La lecture de cet ouvrage permet ainsi d'avoir une première bonne connaissance de travaux récents et originaux et de découvrir, en particulier, l'historiographie anglo-saxonne la plus récente. Lisible par tous, cette genèse livre les meilleurs outils pour découvrir un $\mathrm{XVI}^{\mathrm{e}}$ siècle tellement complexe.

Erica LeHMANN

Denis Crouzet, La Sagesse et le malheur. Michel de L'Hospital, chancelier de France. Seyssel, Champ Vallon, 1998. 15,5 × 24, 608 p., sources et travaux (Époques).

Imposant et empesé par la statuaire académique du XIX ${ }^{\mathrm{e}}$ siècle, Michel de L'Hospital monte à jamais la garde au pied du Palais-Bourbon. Il y scrute de son œil de pierre le pont et la place dits de la Concorde, guettant à travers le flux des voitures un nouveau 6-février, ou un second 21-janvier... L'image historiographique du chancelier est aussi pétrifiée, et conventionnelle, encore que fort contradictoire; cet homme d'État respectable et voué à l'échec est-il venu trop tôt ou trop tard? Fut-il un catholique sincère, un protestant caché, un sectateur de la seule raison d'État, ou un prophète de la déesse Raison? 
Derrière l'image, il fallait chercher l'homme. Robert Descimon avait entamé cette démarche en présentant une réédition de quatre harangues politiques du chancelier (Michel de L'Hospital, Discours pour la majorité de Charles IX..., Paris, Imprimerie nationale, 1993). Denis Crouzet s'y emploie aujourd'hui dans un de ces gros ouvrages dont il a le secret, et avec le trousseau des clefs d'analyse qu'il a forgées dans ses précédents livres : le primat du religieux au $\mathrm{XvI}^{\mathrm{e}}$ siècle et son a-modernité, la violence sacrale, les conceptions opposées du temps vécu, les notions de virtuel et d'imaginaire, le rôle des masques dans la politique-ballet... Avec aussi une connaissance intime des écrits du chancelier, en prose et en vers, en latin et en français, et (est-il besoin de le préciser?) de tous les courants intellectuels, politiques et philosophiques qui traversent alors l'Europe de Rome à Édimbourg. Après avoir étudié tant de violents (du chevalier Bayard aux moines ligueurs), l'historien scrute aujourd'hui le champion de l'antiviolence (qui n'est pas à confondre avec l'actuelle non-violence).

L'entreprise de Crouzet n'est pas et ne se veut pas une biographie anecdotique ou intellectuelle de L'Hospital, mais « une manière d'introspection externe, dont il faut avouer d'emblée toute l'artificialité ». Parti pris tout à la fois audacieux et prudent! Qui va de pair avec la pauvreté des sources sur le personnage même, et la volonté de celui-ci de n'exister que comme homme public (aux yeux des hommes, du moins).

Libre à nous de spéculer sur l'expérience initiale que fut pour ce grand commis de la royauté et ce fervent de l'amitié la loyauté-trahison de son père, fidèle du connétable de Bourbon contre François $1^{\mathrm{er}}$. Ou l'expérience de tolérance intellectuelle et cultuelle qu'il connut quand il étudia à Padoue, juste après la mort de Pomponazzi. Crouzet s'en tient au corpus des œuvres signées par l'auteur (mais pas toujours imprimées de son vivant), et aux grands édits dont il est l'inspirateur et le commentateur, sinon le rédacteur.

Le vécu de L'Hospital n'apparaît que s'il fut pour lui occasion d'une méditation morale qu'il rédige en vers latins ou en prose française (qu'il s'agisse d'une tempête subie sur le Pô en crue, ou de la quête d'une nourrice pour allaiter son petit-fils). Car la source étudiée nous mène au cœur religieux d'un homme du XvI e siècle. Au lieu de voir l'homme privé épaissir et éventuellement expliquer l'homme public, on voit dans ce livre l'homme intérieur sous-tendre et animer l'homme politique.

L'Hospital est défini par Crouzet comme tenant d'un "évangélisme cicéronianiste ». Il est avant tout un chrétien fervent. Peu porté sur les controverses dogmatiques (manifestations de l'orgueil humain et sources de conflits inhumains). Il médite inlassablement sur les malheurs individuels et collectifs (les pires étant les violences commises au nom de la religion). Il voit dans les malheurs autant de signes des temps, signes tout à la fois de l'ire de Dieu et de sa bonté. Mais il ne se place pas pour les interpréter hors du continu temporel, à l'instant du Jugement comme les papistes, à l'instant indépassable de l'Incarnation comme les huguenots. Il vit le temps «en une continuité longue » où le malheur est appel à la confiance, à l'espérance austère mais drue en une «possible bonification du présent », une «bonification des hommes », miracle auquel coopèrent le libre arbitre des hommes et la providence de Dieu.

L'Homme est fragile, fini et faillible. S'il en a conscience (et le malheur a ici vertu pédagogique), s'il ne se prend pas pour un surhomme, il est apte à aider 
l'Homme-Dieu dans l'entreprise du salut. C'est dans cette perspective qu'il y a un bon usage de la politique, un rôle positif des magistrats et des rois : «La politique est un outil du sacré. » Ainsi, une législation n'est pas juste parce qu'elle reflète l'idée éternelle de Justice. Elle l'est parce qu'elle donne dans une conjoncture déterminée les moyens nécessaires pour qu'un groupe humain concret accède à la justice.

C'est dans cette perspective qu'il convient d'interpréter l'édit de tolérance de janvier 1562. Le roi n'est divin et absolu que dans la mesure où il assume pleinement sa finitude d'homme (L'Hospital est bien loin du néoplatonisme cher aux Valois). Le roi n'a pas à choisir entre deux religions qui se veulent exclusives. Il n'a pas non plus à être un «moyenneur», mais un médiateur, un modérateur. Un compromis médiocre serait une solution dépourvue d'inspiration divine, alors qu'il a vocation à donner à la société les moyens de reconstituer son tissu et à Dieu de lui rendre l'harmonie de la foi. Pour cela, il doit user de ruse et de dissimulation (comme le Christ lui-même dans sa prédication) : ne condamnant personne, ne donnant tort à personne, mais garantissant à tous la sécurité, il apaise les rancœurs, il désarme les antagonistes, il rétablit la moralité dans la Cité, il contribue au retour dans les âmes de l'espérance et de la charité sans lesquelles il n'y a pas de foi. Et la «vraie religion » sera alors à la fois restaurée et réformée.

Cette présentation est très schématique et arbitrairement réductrice. Peut-il en être autrement pour un livre dont le défaut essentiel est une trop grande richesse d'information et d'analyse? On doit regretter trop de développements longs, savants et fins sur l'attitude intellectuelle, morale et politique de tel ou tel. Certes, l'auteur s'emploie régulièrement à justifier la présence de ces développements par l'idée que les attitudes proches ou antithétiques de celles du chancelier aident à cerner la sienne propre. Mais il n'arrive pas toujours à convaincre de leur utilité ici (indépendamment de leur qualité intrinsèque). Ainsi, on comprend pleinement la place importante dévolue à Cicéron (et aux feux croisés des lectures qu'on en fait au $\mathrm{XvI}^{\mathrm{e}}$ et $\mathrm{au} \mathrm{Xx}^{\mathrm{e}}$ siècle). Ou celle d'Érasme (et on mesure à ce propos le chemin parcouru depuis le maître livre de Marcel Bataillon) : il s'agit moins de mesurer l'influence du penseur de Rotterdam sur un lecteur français d'une autre génération que d'approcher la pensée profonde et pas toujours explicite ou simple de l'un par la formulation exemplaire de l'autre, en tant que membre éminent de la même famille spirituelle. On admet le rapprochement avec Rabelais (éloigné par le statut social et le registre d'expression, mais voisin par les convictions et peut-être par le vécu d'un même événement). On l'apprécie hautement pour Machiavel, le machiavélisme et l'antimachiavélisme, car il y a là un débat politique et moral essentiel du temps, et Crouzet a bien discerné que le Chancelier y a une place capitale même s'il n'en est pas l'un des protagonistes reconnus. Mais les pages consacrées à l'évangélisme de Marguerite d'Angoulême, à la pensée et à la pratique politique de Calvin et de Théodore de Bèze, et bien d'autres? Et les notes supposées infrapaginales et qui remplissent parfois de leurs caractères serrés plusieurs pages entières : consacrées à l'interprétation allégorique d'un tableau de François Clouet, ou à l'histoire complexe d'une possédée du diable, à une interprétation contemporaine de la SaintBarthélemy, ou à la stigmatisation de la société de cour dans la Soltane de Bounin? Ce sont autant d'articles ou de notices qu'on lirait ailleurs avec le plus grand intérêt, mais qui n'enrichissent pas vraiment l'économie de ce livre. 
Il est un autre thème qui pourrait encore plus être considéré comme hors de propos. Ce sont les pages (p. 456 sqq.) où Crouzet médite sur la méthode historique et sur son métier d'historien. Critiquer la pertinence de la présence ici de telles pages serait ne rien comprendre au livre lui-même. Car ces pages sont le cœur même de l'ouvrage, sa raison d'être, et d'être ce qu'il est. Entreprendre cette histoire «virtuelle » d'un juriste humaniste qui pratiquait une pensée «paradoxique » dans une société où régnait la dissimulation et un univers d'intertextualité triomphante, relève de la mission impossible. La vouloir, et la mener à bien supposent une patience passionnée à combiner des fantasmes cohérents. Cette histoire fabulée est aussi une confession véridique. Et l'historien ne rend (ou ne prête) vie au Chancelier que s'il lui donne de sa vie. S'il reconnaitt (ou établit?) une ressemblance, une parenté entre son héros et lui-même. Et, à l'accent, on se demande parfois lequel des deux est le porte-parole de l'autre. Ainsi dans l'ultime phrase qui clôt La Sagesse et le malheur : «L'Histoire est toujours, par-delà le malheur, par-delà toutes les atrocités et tous les crimes, espérance. »

François BiLLACoIs

Brigitte Віот, Barthélemy Aneau, régent de la Renaissance lyonnaise. Paris, Honoré Champion, 1996. $15 \times$ 23,5, 540 p., bibliogr., index, ill. (Bibliothèque littéraire de la Renaissance, 33).

Barthélemy ANEAU, Alector ou le coq. Histoire fabuleuse. Éd. par Marie-Madeleine Fontaine. Genève, Droz, 1996. 11,5 × 18, cxxvil-312 p. et 699 p., index, bibliogr. (Textes littéraires français, 469).

Il est intéressant de noter une concomitance de publications sur Barthélemy Aneau, connu comme régent du collège de la Trinité (voir le livre de Georgette de Groër, Réforme et Contre-Réforme en France. Le collège de la Trinité à Lyon, Paris, 1995, p. 29-39). Le projet de Brigitte Biot est de réhabiliter un lettré de la Renaissance lyonnaise qui, après avoir été sauvagement assassiné par la foule en tant qu'hérétique en 1561, s'est vu relégué par la postérité au rang de «rimailleur» de bas étage, fermé aux innovations de ses contemporains. Après avoir présenté sa vie, elle étudie l'œuvre du dramaturge, poète, traducteur, échotier et romancier que fut Aneau. Cette démarche est fort éloignée de celle de Marie-Madeleine Fontaine qui affirme dans son introduction à Alector que «la vie de Barthélemy Aneau manque assurément d'intérêt pour un lecteur du $\mathrm{Xx}^{\mathrm{e}}$ siècle » et préfère se consacrer à l'édition de son grand ouvre, Alector ou le coq.

Les deux auteurs divergent d'ailleurs sur d'autres points. Le livre de Biot s'ouvre sur les « Visages et décors d'une jeunesse méconnue » (chap. I). Né à Bourges dans une famille modeste en 1505 selon Biot (en 1510 selon Fontaine, p. Cv-CvI), Aneau reçoit cependant une éducation très soignée, entre à l'université de sa ville natale et 
y aurait suivi les cours d'André Alciat dont il a traduit ensuite les Emblemata. Fontaine affirme, quant à elle (p. CVI-CVII), que l'élogieux accueil d'Aneau au célèbre professeur à Bourges ne prouve pas qu'il en a suivi les cours.

Le chapitre II de la thèse de Biot (p. 55-67) est consacrée à un exposé sur le Lyon commercial et industriel de la première moitié du XvI ${ }^{\mathrm{e}}$ siècle. La présentation des différents cercles humanistes lyonnais tente de circonscrire les milieux dans lesquels Aneau a évolué, ce dernier ayant laissé peu de témoignages de ses amitiés. Ainsi, dans les ateliers d'imprimeurs, en particulier dans celui de Gryphe, Aneau croise Étienne Dolet, Guillaume et Maurice Scève, Clément Marot, François Rabelais, et Biot tente de déterminer la qualité de leurs liens avec le futur régent et éventuellement leur influence sur son œuvre (ce qu'elle reprendra dans les deux dernières parties de son étude). Poursuivant une même ambition, Fontaine, dans sa «biobibliographie » (p. 857-934), livre beaucoup d'informations : y sont retenus « les éléments biographiques induits des travaux publiés du vivant d'Aneau », et ce dans l'ordre chronologique de leur publication.

Le chapitre IV de la thèse de Biot propose un commentaire du projet pédagogique d'Aneau (fourni intégralement en annexe p. 457-461), devenu régent du collège de la Trinité, tout en en soulignant les parentés avec celui d'Érasme. L'auteur en conclut qu'un pédagogue aussi «merveilleusement libéral » qu'Aneau ne peut être un «piètre pédant». Cette première partie biographique se clôt sur le portrait d'Aneau notable lyonnais : Biot répond par l'affirmative mais sans le prouver à la question débattue de la participation d'Aneau à l'organisation de l'Entrée d'Henri II dans la ville. Fontaine la suit sur ce point.

La deuxième partie a pour objet de démontrer l'ouverture d'esprit d'Aneau en tant qu'auteur littéraire, bien que ce dernier n'ait pas cherché à comprendre l'ambition du jeune Joachim Du Bellay et ait préféré critiquer sa Défense et illustration de la langue française (1549-1550) en se concentrant sur des points de détail (p. 225254). Pour sa première œuvre théâtrale, Aneau adopte la forme médiévale du mystère, tout en la modifiant. Il crée ainsi une œuvre inclassable qui n'aura pas de postérité. Cette pièce, le Chant natal (1539), est également une œuvre musicale fort influencée par le style de Clément Marot et sa pratique du contrafactum. Comme Marot et les poètes « évangéliques » (Marguerite de Navarre, Eustorg de Beaulieu), Aneau utilise des airs populaires mais en modifie les vers pour leur donner un sens sacré : «La fermeté de nous deux tant aimée » devient « Nativité de nous tant réclamée ». Faire une pièce en musique, à une époque où cet art est peu estimé sinon par Ronsard, Maurice Scève ou encore les néoplatoniciens tel Marsile Ficin qui prescrit la musique pour chasser le désordre de l'âme, témoigne d'une certaine indépendance. Avec sa seconde pièce, Lyon Merchant (1541), Aneau souhaite ressusciter un genre dramatique de l'Antiquité, la «satyre ». Il préfigure donc l'idéal du poète selon Du Bellay. Cependant, personne ne l'a suivi dans cette entreprise de restauration. Biot détecte dans cette pièce, traitant des grands événements européens depuis 1524 et constituant un éloge de Lyon et du parlement de Paris, une première manifestation de l'idéal politique d'Aneau (une monarchie pondérée) qui évolue vingt ans plus tard dans Alector en rêve d'une démocratie à l'antique (p. 210). Une étude de l'amélioration de la qualité de la poésie d'Aneau entre 1549 et 1552 achève cette deuxième partie. 
Enfin, la dernière partie de la thèse veut éclairer la nature de l' «hérésie » d'Aneau. En premier lieu, son adoption du contrafactum aurait conduit certains à le situer dans le camp des « réformés ». D'autre part, Biot affirme qu'Aneau est considéré hérétique dans les années 1560 , bien qu'il ne renie pas l'orthodoxie, parce qu'il a des préoccupations mystiques nourries par les écrits des philosophes et des alchimistes. Biot éclaire les motivations de ceux (la foule lyonnaise, les Jésuites) qui ont fait d'Aneau un «réformé », en invoquant la rivalité avec les membres de la compagnie de Jésus qui sont parvenus en 1565 à prendre la direction du collège de la Trinité. Et finalement, en étudiant la mystique d'Aneau telle qu'elle s'offre dans Alector, l'auteur trace le portrait d'un homme peu concerné par les débats de ses contemporains sur la façon de vivre sa foi. Fontaine souligne le caractère quelque peu anachronique du personnage : Aneau « se croyait encore à l'apogée de la pédagogie humaniste, celle où l'on pensait pouvoir généraliser tranquillement l'enseignement d'un Ficin ou d'un Politien ».

Ces deux travaux viennent ainsi à point pour compléter la bibliographie concernant Aneau. L'édition critique d'Alector facilitera la poursuite et l'approfondissement des recherches sur ce romancier si longtemps négligé.

Erica LeHMANN

Monluc, d'Aubigné, deux épées, deux plumes. Actes du colloque «Monluc, d'Aubigné. Les événements en Aquitaine après 1560 », organisé par la Société française d'étude du seizième siècle et le centre Matteo-Bandello d'Agen, tenu à Agen, Estillac, Casteljaloux, Nérac, les 4-6 octobre 1996, textes réunis par Jean Cubelier de Beynac et Claude-Gilbert Dubois, av.-pr. de Marie-Thérèse François-Poncet, postf. de Madeleine Lazare. Agen, Centre Matteo-Bandello, 1999. $15 \times 21,296$ p., index.

Ce colloque a permis de réunir plusieurs communications consacrées aux guerres de Religion en Aquitaine et à deux capitaines, l'un catholique, l'autre protestant, qui passèrent de longues années à guerroyer entre le Poitou et les Pyrénées. Ces régions furent en effet le théâtre de conflits extrêmement violents, car la communauté réformée y était nombreuse et disposait d'appuis solides, dans la noblesse et parmi les notables urbains. La lutte y fut militaire, bien sûr, mais aussi intellectuelle. Elle se poursuivit d'ailleurs avec âpreté dans les ouvrages qui parurent au cours du XVII siècle, par exemple dans Les Antiquitez d'Agen, qui furent publiées en 1605 par Jean Darnalt. Michel Cassan souligne en effet que cet avocat au parlement de Bordeaux consacra de longues pages à exalter la foi chrétienne pour mieux condamner les hérésies, notamment le calvinisme. En dépit de l'édit de Nantes et de son application rigoureuse sous le règne d'Henri IV, les blessures confessionnelles étaient toujours béantes dans le quart Sud-Ouest du royaume.

À première vue, tout oppose le Gascon Blaise de Monluc (1502-1577) et le Saintongeais Agrippa d'Aubigné (1552-1630). Madeleine Lazare rappelle que Monluc appartenait à une famille de noblesse désargentée, et qu'il n'eut pas la chance de 
bénéficier d'un précepteur. D'Aubigné, au contraire, reçut une éducation très soignée : dès l'âge de six ans, il lisait « aux quatre langues » grâce à des maîtres formés à l'humanisme le plus raffiné. Monluc était fermement attaché au catholicisme, par goût personnel, par tradition familiale et par respect pour l'autorité, tandis que d'Aubigné avait été élevé par son père dans la conviction qu'il devait lutter pour la Réforme et instaurer ici-bas le royaume de Dieu en faisant si besoin le don de sa vie. Pourtant, au-delà de ces divergences, les similitudes entre les deux personnages sont nombreuses. Ils nourrissaient notamment une véritable passion pour la guerre, à laquelle ils s'étaient initiés dès l'adolescence. Robert Knecht montre ainsi que Monluc expérimenta de nouvelles techniques de fortification; il construisit par exemple plusieurs remparts en terre, qui étaient destinés à réduire l'impact des boulets adverses. De même, il prit toujours soin d'observer attentivement les lignes ennemies avant de lancer un assaut, afin d'exploiter au mieux leurs faiblesses. De son côté, d'Aubigné joua un rôle décisif pendant les campagnes des années 1577-1584, qu'il décrivit avec force détails dans son œuvre. S'il fit preuve de beaucoup d'habileté pour se mettre en valeur, il représenta néanmoins, selon Gilbert Schrenck, un atout précieux pour le roi de Navarre, qui sut utiliser au mieux ses qualités de stratège.

Tous deux eurent conscience qu'ils vivaient des événements exceptionnels. Chacun à leur façon, ils cherchèrent à les raconter afin d'en conserver la trace pour édifier les générations futures. Anne-Marie Cocula démontre toutefois que les Commentaires de Monluc ne visaient pas le même objectif que L'Histoire universelle de d'Aubigné. En effet, le premier se posait avant tout comme un mémorialiste, alors que le second faisait œuvre d'historien. Le capitaine catholique, qui avait été accusé des pires violences, tenta un plaidoyer pro domo, tandis que le protestant choisit d'écrire son livre lentement, pendant une vingtaine d'années, afin qu'il pût être lu pendant des siècles à l'avenir. Monluc s'inscrivait donc dans une actualité encore brûlante, alors que d'Aubigné bénéficiait déjà d'un certain recul. Pourtant, leur rapport à la violence n'était pas très différent. Pour James J. Supple, Monluc était proche de La Boétie, car il redoutait comme lui tous les désordres nés des troubles religieux. Enfant de notable, proche des princes huguenots, d'Aubigné n'avait, lui non plus, rien d'un révolutionnaire : pour lui, la violence devait simplement être utilisée à bon escient, afin de faire triompher le règne de Dieu tout en respectant certaines règles. Sa pensée peut ainsi être comparée à celle de François de La Noue, autre grand capitaine protestant, qui exalta dans ses Discours les guerres d'Italie pour mieux dénoncer la sauvagerie inédite des guerres de Religion. Le récit de la guerre permettait donc d'affirmer sa conception du monde, tout en inscrivant son action personnelle dans le dessein de Dieu. Chez Monluc, la narration possédait une triple vertu: elle racontait un haut fait d'armes, elle présentait l'auteur sous son meilleur jour et démontrait la justesse de sa cause. À titre d'exemple, Claude-Gilbert Dubois analyse un épisode de 1569 , au cours duquel un moulin à la dérive, sur la Garonne, détruisit un pont de bateaux qui avait été bâti par les protestants. Pour Monluc, cet événement n'était dû ni au hasard, ni à la chance : il prouvait simplement que Dieu était aux côtés des catholiques pour les aider à balayer l'hérésie comme un fétu de paille. Même si d'Aubigné tendait plutôt à inscrire les guerres de Religion dans une vaste Histoire universelle, il n'oublia pas, lui non plus, de narrer ses propres aventures. Il fit ainsi dire à son imprimeur «qu'ayant commencé son premier siège dans Orléans en 1562, et esté soldat 54 ans, capitaine 50, mestre de 
camp 44, et mareschal de camp 32 années, [...] il auroit esté trop lasche ou trop malheureux s'il n'avoit à respondre en son nom de plusieurs exploicts ». Comme chez Monluc, l'écriture était donc, pour d'Aubigné, un moyen de parler à la première personne, de réaffirmer son engagement et de le justifier aux yeux de Dieu et des hommes.

Dans cette perspective, nos deux capitaines étaient sans doute inspirés par la vie de Bayard, dont les exploits avaient été écrits et publiés dès 1527 par Symphorien Champier. Pour les catholiques comme Monluc, le chevalier sans peur et sans reproche avait prouvé que l'homme était capable de faire son salut par lui-même, en joignant les œuvres à la foi. Mais pour les protestants comme d'Aubigné, Bayard était également, depuis un demi-siècle, un modèle concentrant en sa personne toutes les vertus nobiliaires. La geste de Bayard en Italie, qui connut un grand succès dans toute la noblesse, inspira donc les gentilshommes qui, comme eux, cherchaient des repères parce qu'ils se sentaient engagés dans une croisade contre les faux dogmes. Ce colloque, qui présente à la fois leurs violences et la représentation qu'ils s'en faisaient, nous invite donc à multiplier les comparaisons entre tous les capitaines des guerres de Religion, catholiques ou protestants, qui écrivirent des Mémoires ou dont les faits et gestes furent racontés par leurs contemporains.

Laurent BouRQuIN

Olivier Poncet, Pomponne de Bellièvre (1529-1607). Un homme d'État au temps des guerres de Religion. Préf. Bernard BARBiche. Paris, École des chartes, 1998. $16 \times 24$, xiv-490 p., tabl., pl., bibliogr., index (Mémoires et documents de l'École des chartes, 50).

Comme le fait remarquer Bernard Barbiche dans sa préface, Pomponne de Bellièvre n'est généralement mentionné dans les manuels d'histoire que parce qu'il s'opposa à Sully, entre 1602 et 1604, à propos de la création de la paulette. Or la thèse d'Olivier Poncet vient légitimement tirer de l'oubli ce personnage, qui eut une très longue carrière et joua un rôle majeur dans le gouvernement royal au cours de la deuxième moitié du Xvi siècle.

Né en 1529, il était le fils cadet de Claude Bellièvre, un brillant juriste issu d'une famille de notaires lyonnais, qui avait étudié à Pavie et à Turin. Conseiller de sa ville en 1523-1524 et 1528-1529, Claude connut une belle ascension en devenant l'avocat du roi en la sénéchaussée de Lyon et bailliage de Mâcon (1532), procureur général au parlement de Grenoble (1536) et premier président de ce parlement (1541). Du côté de sa mère, Louise Faye, on trouvait également de solides magistrats, comme Barthélemy Faye, l'oncle de Pomponne, qui exerçait une charge de conseiller au parlement de Paris. Ces appuis familiaux furent décisifs au début de sa carrière lorsqu'il fut nommé, le 27 juin 1554, conseiller au parlement de Chambéry. Comme son père et son oncle, Pomponne de Bellièvre était donc d'abord un grand magistrat, dont la compétence juridique fut le premier atout.

Son itinéraire le conduisit pourtant loin de la magistrature, car il se mit à exercer assez tôt des charges diplomatiques et financières. Pour Poncet, le personnage était 
d'une exceptionnelle polyvalence, assez singulière à son époque pour attirer l'attention du roi de France. En 1557, il contribua ainsi à pacifier une révolte qui avait été attisée dans sa région par Philippe II. Trois ans plus tard, il fut nommé lieutenant général du présidial de Laon, c'est-à-dire le chef de la justice dans une province frontalière sensible, où le maintien de l'ordre était crucial pour assurer la protection de l'Île-de-France. En 1564, il fut envoyé en Suisse pour être l'ambassadeur du roi de France auprès des cantons, afin d'y recruter des mercenaires et de distribuer de l'argent aux Ligues.

C'est au cours des années 1560 que Pomponne de Bellièvre sut capter la confiance de Catherine de Médicis, à tel point qu'elle lui demanda en 1573 d'accompagner comme conseiller le duc d'Anjou, qui venait d'être élu roi par la diète polonaise. Ce séjour en Pologne fut sans doute une étape très importante dans sa carrière, car il fit partie des quelques fidèles qui restèrent auprès du futur Henri III de bout en bout, jusqu'à son retour en France, à la mort de Charles IX en 1574. Après avoir gagné la confiance de la reine mère, Pomponne sut donc gagner celle du jeune souverain, qui l'envoya en mission diplomatique dans le Palatinat (1576), en Languedoc et en Guyenne (1581). Dès le début du règne, il fut choisi pour être son surintendant des finances, en 1574. Cette charge était une grande nouveauté car, à l'époque de Charles IX, cette administration relevait directement du Conseil des finances, créé en 1563. La nomination de Pomponne de Bellièvre comme surintendant traduisait donc un souci d'efficacité très neuf : Henri III entendait placer là un homme de confiance non seulement parce qu'il s'agissait d'un poste stratégique, mais aussi parce qu'il voulait en prendre lui-même les commandes. La collaboration du roi et du surintendant fut donc très étroite, à tel point qu'il reste difficile, selon Poncet, «de déterminer l'influence réelle de Bellièvre dans les orientations financières du règne ».

Les attributions que l'on donnait à Pomponne l'éloignaient ainsi des carrières juridiques, même s'il occupa, de 1576 à 1580, la présidence du parlement de Paris. Sans forcer le trait, on peut donc considérer que dans la deuxième moitié des années 1570, il fut à la fois et en même temps diplomate, financier et magistrat... Pourtant, Pomponne de Bellièvre connut une brutale disgrâce en 1588. Après la journée des Barricades du 12 mai, Henri III perdit en effet le contrôle de sa capitale et tenta de reprendre l'initiative en convoquant les États généraux. Afin d'offrir aux députés un gage de sa bonne foi, il décida, la mort dans l'âme, de se séparer de ses plus anciens conseillers, comme Cheverny, Villeroy, Brulart et Bellièvre. D'après Poncet, ce départ traduisait toutefois un substantiel changement de ligne politique, car Bellièvre ne fut pas rappelé après l'assassinat des Guises et la clôture des États.

Il connut ainsi une longue traversée du désert, qui ne s'acheva qu'en 1592 : à cette époque, il prêta en effet sa plume à Henri IV pour réclamer dans un libelle la levée de l'excommunication pontificale qui pesait sur le monarque. Au cours de l'été 1593, il récidiva en publiant un Advis aux Français qui justifiait la conversion du roi au catholicisme. Henri IV apprécia donc rapidement les multiples talents de Bellièvre, qui fut envoyé à Lyon en juin 1594 afin de s'assurer de la fidélité des notables qu'il connaissait si bien. En novembre 1594, il entra dans le nouveau Conseil des finances, et put incarner avec quelques autres une certaine continuité du personnel politique entre les Valois et les Bourbons. C'est à la mort de Cheverny, en 1599, que Pomponne de Bellièvre connut la consécration suprême : il le remplaça 
comme chancelier et devint l'un des premiers personnages du gouvernement, aux côtés de Sully, qui se chargeait quant à lui des finances.

En tant que chancelier, Pomponne de Bellièvre s'en tint à une ligne extrêmement prudente, à tel point qu'il fit taire ses convictions personnelles pour soutenir les mesures qui lui semblaient les plus raisonnables. Même s'il était, par exemple, favorable à la publication officielle des décrets du concile de Trente, il n'alla pas jusqu'à la réclamer parce qu'il était convaincu que le gallicanisme était trop puissant dans le pays, et que les décisions tridentines ne seraient jamais acceptées par le clergé et les juges. De même, s'il était personnellement hostile aux Jésuites, il préféra ne pas s'opposer à leur retour en 1603. Bellièvre agit ainsi en loyal serviteur de l'État, jusqu'à recueillir les témoignages à charge dans le procès Biron, en 1602. Mais à cette époque, il commença à s'opposer de plus en plus nettement à Sully, en particulier au sujet de la fameuse paulette, cette taxe que le surintendant des finances envisageait de faire payer aux officiers pour les autoriser, en échange, à transmettre librement leur charge à leurs héritiers. Pomponne de Bellièvre y était violemment hostile, parce qu'il estimait que cette mesure empêcherait à l'avenir le roi de choisir librement ses collaborateurs. Toute suppression d'office serait d'ailleurs impossible, puisqu'elle supposerait de rembourser les propriétaires. Il pensait aussi qu'ils seraient fatalement moins compétents, car leur fortune serait un critère déterminant dans leur carrière. Il prédisait même une augmentation du prix des charges, qui deviendraient si chères qu'elles ne pourraient plus être achetées que par des spéculateurs, excluant les familles de magistrats traditionnels. Ses arguments ne furent pas entendus par le roi, qui préféra suivre les avis de Sully en décembre 1604. Cet échec précipita sa chute définitive : il fut progressivement mis à l'écart, et en octobre 1605, le roi décida de confier les sceaux à Nicolas Brulart de Sillery. Pomponne de Bellièvre mourut deux ans plus tard, le 9 septembre 1607.

Plutôt que de tracer un portrait culturel, politique ou religieux de Pomponne de Bellièvre, Poncet a préféré insister sur son parcours social. Grâce à un patient travail de collecte des archives au département des Manuscrits de la Bibliothèque nationale et au Minutier central, il a mis en évidence le réseau familial et les protections dont le héros de son livre sut profiter tout au long de sa vie. Son oncle, Barthélemy Faye, était en effet un ami personnel de Michel de L'Hospital, qui joua peut-être un rôle dans sa nomination au présidial de Laon, en 1560, et dans son envoi en Suisse, en 1564. Il fut ensuite protégé par Jean de Morvilliers, à qui Catherine de Médicis confia les sceaux en 1568, après la disgrâce de L'Hospital. Plus tard, il fut soutenu par les membres de sa famille au cours des difficiles années 1588-1592. Porté par les espoirs de tout un clan, Pomponne fut ainsi aidé par son cousin Jacques Faye, président à mortier au parlement de Paris transféré à Tours.

Protégé par tout un réseau familial et amical, Pomponne de Bellièvre sut également faire profiter les autres de sa réussite. Dès le milieu des années 1560, il devint par exemple l'ami de Jean Grangier, le trésorier des ligues suisses, qui fut nommé grâce à lui ambassadeur en Suisse, puis maître d'hôtel ordinaire du roi et secrétaire de la Chambre. Pomponne de Bellièvre entretenait d'excellents rapports avec des protestants comme Jean et Jacques de La Fin, deux huguenots du Bourbonnais qui vivaient dans l'entourage de François d'Anjou. En 1575, ils permirent de négocier la paix avec le prince de Condé, et en échange, ils obtinrent des terres de la part du roi de France. Bellièvre intervint même en personne pour qu'ils puissent recevoir 
d'autres récompenses. Du côté catholique, il sut également conserver de bonnes relations avec des prélats intransigeants, comme Pierre d'Épinac, qui fut archevêque de Lyon entre 1573 et 1599 . Il alla même jusqu'à louer sa résidence parisienne, en mars 1589, à deux ligueurs, Étienne Gallinet et Denis de Roissieu, tous deux commissaires et intendants généraux des vivres et armées de la Ligue, pour éviter que sa demeure ne fût pillée après la mort des Guises.

Cette dernière anecdote permet de mettre en évidence le pragmatisme de Pomponne de Bellièvre, qui ne s'attardait pas sur la confession de ses interlocuteurs lorsqu'ils pouvaient lui être d'une quelconque utilité. Il était pourtant très attaché à la religion de ses pères, convaincu que les guerres civiles étaient néfastes pour tout le royaume. Il proposa même, en 1586, d'organiser une grande croisade contre les Turcs afin de réconcilier les Français, les Anglais et les Espagnols, quelles que fussent leurs confessions. Mais sur le plan politique, Pomponne de Bellièvre restait préoccupé avant tout par la stabilité de l'État et son autonomie. L'homme était donc un vrai «politique », proche de la pensée de Michel de L'Hospital telle qu'elle a été restituée par Denis Crouzet dans La Sagesse et le malheur (Seyssel, 1998).

Laurent BOURQUIN

Coexister dans l'intolérance. L'édit de Nantes, 1598. Éd. Michel GrandjEan et Bernard Roussel, collab. François Bos et Béatrice Perregaux Allisson. Genève, Labor et Fides, 1998. $15 \times 23,544$ p., index (Histoire et société, 37).

Pour une large part fruit d'un enseignement donné dans les facultés de théologie des universités de Suisse romande, ce recueil de vingt-six contributions est un apport de premier ordre aux débats historiographiques suscités par l'interprétation de ce texte majeur qu'est l'édit de Nantes. Son titre indique la rupture opérée avec une lecture traditionnelle ayant érigé l'Édit en acte fondateur de la tolérance, au prix d'une méconnaissance ou d'un aveuglement sur la signification effective de cette notion par les hommes du $\mathrm{XvI}^{\mathrm{e}}$ siècle.

Les contributions ont été distribuées en six chapitres, envisageant successivement la date de l'Édit rapportée de façon démonstrative au 30 avril et non au 13 par Jean-Louis Bourgeon, sa rédaction, son contexte, ses interprétations et son historiographie. Le dernier volet s'écarte de cette trame pour inscrire le texte dans son environnement européen.

Olivier Christin rappelle combien l'édit de Nantes n'est qu'une des nombreuses paix de religion proclamées au $\mathrm{XVI}^{\mathrm{e}}$ siècle. En effet, confrontés à l'éclatement confessionnel de leurs territoires, les princes et leurs juristes ont inventé les règles d'une cohabitation pacifique de leurs sujets. Avec les paix de Cappel (1531) ou d'Augsbourg (1555) analysée par Bernard Vogler, ils ont promu les notions du bien commun, de l'intérêt de l'État, de la Res publica, qui se retrouvent dans l'édit de Nantes. Des débats accompagnant chaque publication de paix comme des actes de Lazarus von Schwendi dans l'Empire ou du chancelier Michel de L'Hospital en France, émerge l'idée de la concorde civile que Claire Grandet retrouve à travers 
l'analyse des fêtes commémoratives de la paix de Westphalie à Augsbourg de 1648 à 1660. Mario Turchetti à partir d'une analyse du libelle De la concorde de l'État restitue l'arrière-plan politique de l'Édit et montre comment Henri IV voulait instaurer la concorde et la paix civile avant d'envisager la réunification religieuse de ses sujets. Marianne Carbonnier-Burkard rencontre ces visées dans les préambules des édits français publiés dans les années 1560 . Un canevas identique, repérable d'un texte à l'autre, développe un argumentaire en trois points. Premièrement, le trouble des consciences individuelles a induit un désordre généralisé dans le pays. Deuxièmement, le roi qui doit protéger ses sujets et leur garantir la paix a décidé après mûre réflexion de recourir à l'Édit. Sa teneur - troisième acte - est insatisfaisante puisque la division religieuse est admise, mais cette réponse douloureuse à une situation de détresse est un moindre mal, préférable à l'état de guerre qu'elle cherche à éteindre. À la condition que chacun obéisse au Prince qui voit son pouvoir théoriquement conforté.

Cette solution en forme de reconnaissance de la confession des réformés a couru 287 mois sur 435 entre 1559 et 1598 . Philip Benedict a établi ces résultats qui suggèrent une cohabitation quotidienne des protestants et des catholiques. Certes, « la saison des Saint-Barthélemy », d'après la formule de Jules Michelet, a montré les limites de cette option, mais l'on peut aussi se demander si cette coexistence provisoire et fréquemment reconduite n'a pas servi d'apprentissage à la tolérance de l'Autre et contribué à l'acceptation de l'édit de Nantes.

L'homme qui le rédigea, Pierre Forget de Fresnes, fait l'objet d'une analyse de Robert Descimon. Il retrace l'ascension lignagère et personnelle de ce secrétaire d'État nommé par Henri III, le passage en trois générations de la marchandise tourangelle au service du Prince. Un mariage tardif ouvre les portes de l'aristocratie et couronne une ascension rapide, comme le $\mathrm{XvI}^{\mathrm{e}}$ siècle en permet. Ce serviteur de l'État a mis en forme un texte élaboré au cours de rudes tractations entre Henri IV et ses anciens coreligionnaires de 1593 à 1598. Un homme domine cette séquence : Philippe Duplessis-Mornay auquel trois articles sont consacrés. Olivier Fatio scrute l'immense érudition du gouverneur de Saumur et rappelle qu'il fut un théologien. Hugues Daussy souligne son rôle d'intermédiaire entre les réformés qui respectent sa science, son attachement à la foi réformée et le roi qui lui accorde depuis longtemps sa confiance et se loue de son pragmatisme et de sa modération. Fidèle au Prince et à la Religion, Duplessis-Mornay est à la fois, ainsi que l'analyse Nicolas Fornerod un homme d'État soucieux de conciliation entre chrétiens et un réformé capable de rédiger des textes prêtant à polémique. Son portrait s'en trouve brouillé, sauf à penser que sa position médiatrice ressort à cette dualité entre sagesse politique et prosélytisme protestant. Ces postures antinomiques lui vaudraient l'attention du roi et la confiance de ses coreligionnaires, toutes deux si précieuses lors des délicates négociations de l'Édit. Le texte, contesté par de grands nobles huguenots « carnassiers » (Janine Garrisson), précède de quelques jours la signature de la paix de Vervins et Béatrice Nicolier procède à un rapprochement heureux des deux décisions imposées par Henri IV. Le roi aurait déclaré la guerre à l'Espagne pour se défaire de la Ligue, desserrer l'emprise de son envahissant voisin mais, une fois vainqueur, être en mesure de contraindre les protestants à accepter ses volontés. La paix de Vervins aurait donc permis à Henri IV d'imposer l'édit de Nantes, à moindres frais, à ses anciens coreligionnaires. 
Péniblement négocié, l'Édit entre en application avec de grandes difficultés. Le cas de la Provence, où le parlement regimbe pendant deux ans (Gabriel Audisio), le montre. S'ouvre alors une autre période de l'histoire de l'Édit et des relations entre catholiques et protestants, brossée par une demi-douzaine de contributions. Gottfried Hammann résume les enjeux de la pleine application de l'Édit auprès de la société, à savoir la nécessaire occultation du passé, l'amnistie-amnésie afin de désamorcer l'agressivité des parties hier adverses et investies d'une coresponsabilité des troubles. Françoise Chevalier, Raymond Mentzer, dans le prolongement de l'analyse des synodes nationaux de 1594-1598 menée par Bernard Roussel, déclinent la médiocre exécution de l'Édit, les difficultés éprouvées par les protestants, les tensions entre les communautés pouvant dégénérer en procès. Marc Venard, Bertrand Haan, Alain Tallon envisagent la réception de l'Édit du côté catholique et romain. Certes, l'Église et la papauté condamnent la décision et vitupèrent contre la tolérance. Mais, à l'image du nonce Santa Croce en France au début des années 1560, l'Église demande la suppression d'un texte qu'elle cherche à utiliser pour organiser ses lignes de défense et de contre-attaque. L'édit de Nantes devient alors un acte fondateur de la reconquête catholique officielle, comme le souligne Venard, en rupture avec le discours historiographique habituel. Seuls François Garrisson en 1950 et Élisabeth Rabut en 1987 avaient signalé cette dimension de l'Édit. David El Kenz, au terme d'une réflexion portant sur l'institutionnalisation de la communauté réformée de 1563 à 1598 interprète l'édit de Nantes comme un carcan. La survie du calvinisme institué est garantie mais sa vigueur et sa vocation universaliste sont contenues et la situation risque d'être une source d'affaiblissement sensible.

À peine révoqué, l'Édit devient objet d'histoire (Hubert Bost). Le pasteur d'Alençon, Élie Benoist exilé à Delft, écrit sa grande œuvre, l'Histoire de l'édit de Nantes. Il veut transmettre le souvenir des réformés et réfuter les allégations déversées sur eux. L'ouvrage voudrait être d'histoire, mais rédigé à chaud, il est parasité par la défense du petit troupeau et Benoist fait de l'Édit rompu un traité conclu entre le roi et ses sujets là où il n'y a qu'un acte de la toute-puissance régalienne. La production intellectuelle de ses successeurs des années 1787-1948, analysée par Patrick Harismendy révèle une forte polarisation parisienne de la production historique. La province est méconnue et le discours brille par un surinvestissement éclatant du politique à côté de la faible part du religieux. Les interprétations de l'Édit surtout véhiculées par des pasteurs-historiens jusqu'au $\mathrm{xx}^{\mathrm{e}}$ siècle portent sur le droit des minorités, l'essence républicaine du protestantisme ou le rapport entre le texte et l'égalité naturelle des hommes devant Dieu. Quant à l'historiographie catholique du XIX ${ }^{\mathrm{e}}$ siècle, elle fait l'objet d'une contribution de Guy Bedouelle, fondée sur l'analyse des prises de position des catholiques face aux célébrations du troisième centenaire de l'Édit. L'auteur distingue les ecclésiastiques qui fustigent l'Édit et ceux qui, reprenant la variation dialectique de la thèse et de l'hypothèse, affirment que l'Édit est une chose affreuse mais qu'en raison des circonstances, il n'était qu'un moindre mal. En somme, l'on retrouve à trois cents ans de distance, les variations du nonce Santo Croce ou du pape Clément VIII qui se déclare crucifié par l'Édit tout en cherchant comment l'utiliser pour contrer le protestantisme.

C'est ce qui eut lieu et conduit Bernard Cottret à parler de la réussite de l'Édit. Le texte a atteint son but avoué : instaurer la coexistence religieuse des catholiques et des réformés; il a aussi atteint un objectif inavoué : faciliter la victoire de la religion 
romaine sur le calvinisme. Ainsi, l'Édit que J.-H. Mariéjol assimilait au porche d'entrée dans l'ère de la tolérance se révèle finalement favorable à l'intolérance. L'intitulé du recueil prend alors tout son sens, mais à condition de rappeler combien l'Édit fut instrumentalisé par les églises catholique et protestante tout au long du $\mathrm{XVII}^{\mathrm{e}}$ siècle. Toutes deux voulaient édifier une société confessionnelle. Leur concurrence était inéluctable et la conflictualité fut vive entre des chrétiens qui ne surent ou ne purent s'opposer aux visées de leurs clergés. Le temps de l'Édit, hier vu avec les lunettes de la tolérance, lu aujourd'hui au plus près des faits, apparaît, selon l'expression d'Élisabeth Labrousse, comme un temps de guerre froide après la guerre chaude.

Michel CASSAN

Tolérance et intolérance. De l'édit de Nantes à nos jours. Dir. Guy SAuPIn, préf. Jean-Marc Ayrault et Edmond Hervé. Rennes, Apogée, Presses universitaires de Rennes, 1998. $22 \times 30,128$ p., ill. en noir et en coul., bibliogr.

L'ouvrage est un exemple réussi des effets croisés de la commémoration historique et du mécénat politique. En effet, la célébration du quatrième centenaire de l'édit de Nantes, l'aide matérielle des municipalités nantaise et rennaise ont permis à Guy Saupin de réunir une équipe d'historiens universitaires et de nous offrir ce livre. Formellement, l'ouvrage est magnifique. Sa mise en page est soignée et accompagnée d'une iconographie abondante puisée à des sources connues tableaux sulpiciens, caricatures de Daumier, premières pages de journaux, mais aussi souvent ignorées comme les vitraux des églises vendéennes. Cette illustration ne se contente pas d'être agréable à regarder. Elle est judicieusement choisie, accompagnée de légendes copieuses et constitue l'un des aspects les plus remarquables du livre. Les huit contributions ont été rangées selon un plan chronologique, articulé autour des dates charnières évidentes, 1598, 1685, 1787, 1789-1791, 1905. D'un chapitre à l'autre, l'ouvrage aborde la thématique de la liberté de conscience, de la pluralité confessionnelle, des choix des pouvoirs politiques vis-à-vis des minorités religieuses et au premier chef, des protestants. Cela conduit à de fréquents et amples dépassements du registre de la tolérance et de l'intolérance et à la narration des événements survenus entre puissances catholiques et protestants du XvI siècle à l'époque contemporaine. Ce choix satisfera les attentes d'un large public éclairé disposant ainsi d'une synthèse close par le dense essai de Jean Baubérot qui replace dans leur épaisseur historique des questionnements contemporains tels que le port de marqueurs confessionnels, le statut actuel des minorités religieuses ou les rapports entre tolérance et liberté(s). On peut regretter toutefois l'absence d'une chronologie commentée des événements et des principaux écrits suscités du XvI ${ }^{\mathrm{e}}$ siècle $\mathrm{au} \mathrm{xx}^{\mathrm{e}}$ siècle par les notions de concorde, tolérance, liberté de conscience ou de culte, mais cette courte réserve ne doit pas nous empêcher d'apprécier un livre clair, solide, de facture classique.

Michel CASSAN 